\title{
Appréhender le développement des organisations à la croisée du cours d'action et de l'approche instrumentale : la perspective transitionnelle
}

Understanding the development of organisations at the crossroads of the course of action and the instrumental approach: the transitional perspective

\section{Anne Bationo-Tillon, Céline Poret et Viviane Folcher}

\section{OpenEdition \\ Journals}

Édition électronique

URL : https://journals.openedition.org/activites/5371

DOI : 10.4000/activites.5371

ISSN : $1765-2723$

Éditeur

ARPACT - Association Recherches et Pratiques sur les ACTivités

\section{Référence électronique}

Anne Bationo-Tillon, Céline Poret et Viviane Folcher, « Appréhender le développement des organisations à la croisée du cours d'action et de l'approche instrumentale : la perspective transitionnelle », Activités [En ligne], 17-2 | 2020, mis en ligne le 15 octobre 2020, consulté le 25 août 2021. URL : http://journals.openedition.org/activites/5371; DOI : https://doi.org/10.4000/activites. 5371

Ce document a été généré automatiquement le 25 août 2021.

\section{cc) $(1) \odot$}

Activités est mis à disposition selon les termes de la licence Creative Commons Attribution - Pas d'Utilisation Commerciale - Pas de Modification 4.0 International. 


\section{Appréhender le développement des organisations à la croisée du cours d'action et de l'approche instrumentale : la perspective transitionnelle}

Understanding the development of organisations at the crossroads of the course of action and the instrumental approach: the transitional perspective

Anne Bationo-Tillon, Céline Poret et Viviane Folcher

\section{NOTE DE L'ÉDITEUR}

Article soumis le 9 mars 2020, accepté le 10 mai 2020

\section{Introduction}

Nous proposons au sein de cet article d'aborder la question des voies conceptuelles possibles pour penser/étudier/accompagner le développement des organisations par l'entremise des formes transitionnelles des activités individuelles et collectives. Accompagner une organisation du travail à devenir ce qu'elle n'est pas encore nécessite d'appréhender le développement comme un pari dont l'issue n'est jamais fixée a priori (Bationo-Tillon, 2017; Bationo-Tillon \& Rabardel, 2015; Folcher \& Bationo-Tillon, 2018). Après avoir introduit le concept de frontières pour définir le développement comme franchissement de frontières, nous prolongerons nos explorations du côté des phénomènes transitionnels pour appréhender les chemins singuliers de développement des individus et des collectifs d'individus. Cependant, pour rendre compte des dynamiques développementales, le chercheur se heurte à la 
difficulté suivante: mondes $^{1}$ du sujet, collectifs, sujets, systèmes d'activités se transforment de concert. Les limites bien établies de ces frontières disparaissent, car il ne faut pas s'y tromper, ce sont bien les relations entre ces entités qui se transforment. Il s'agit donc de se doter de cadres théoriques à même de rendre compte de ce qui émerge dans l'entrelacs des systèmes d'activités. Le cœur de l'article consistera donc à exposer l'hybridation théorico-empirique que nous avons mise en œuvre au sein de deux recherches distinctes pour appréhender des formes transitionnelles de l'activité qui permettent aux sujets et aux collectifs de créer de la continuité entre des systèmes d'activité discontinus. Ainsi, nous retraçons au sein de cet article la manière dont nous avons articulé deux approches conceptuelles issues du champ de l'ergonomie : le cours d'action (Theureau, 2004) et l'approche instrumentale (Rabardel, 1995) pour aborder les questions de développement afin de mettre à jour des instruments transitionnels élaborés par les acteurs engagés dans des franchissements de frontières. Enfin nous discuterons des apports possibles de cette approche dite transitionnelle pour penser les organisations en devenir.

\section{Le développement des organisations : un franchissement de frontières permis par des phénomènes transitionnels}

2 Le développement peut être défini comme franchissement de frontières entre deux systèmes d'activité hétérogènes (Akkerman \& Bakker, 2011). Cependant, cette manière de décrire le développement consiste à adopter le point de vue d'un observateur extérieur. Que se passe-t-il du point de vue de ceux ou celles qui sont engagé.e.s dans ce franchissement de frontières? C'est ce que nous proposons d'approcher grâce à la perspective transitionnelle.

\subsection{Franchissement de frontières et développement des organisations}

3 Pour Akkerman et Bakker (2011), les frontières sont fonctionnellement ambivalentes puisqu'elles permettent de relier les composantes d'un système tout autant que de les diviser et les séparer. Les frontières sont dans ce contexte, des différences socioculturelles qui produisent de la discontinuité dans les actions et les interactions d'individus et/ou de groupes d'individus. Par ailleurs, ces auteurs s'intéressent aux personnes-frontières qui œuvrent aux frontières, construisant des ponts entre les mondes, entre les pratiques, entre les systèmes d'activité. Ces personnes-frontières se confrontent à la dialectique du proche et du lointain, puisqu'elles agissent comme des ponts entre des mondes séparés, tout en représentant et incarnant la division de ces mondes relatifs. Tantôt considérées, comme des maillons indispensables permettant d'entrelacer une diversité de pratiques (Wenger, 1998). Tantôt considérées comme des personnes périphériques, n'appartenant et ne participant réellement à aucune communauté de pratiques.

4 La frontière permet de délimiter ce qui est déjà institué au sein d'une organisation, d'une communauté, de ce qui n'est pas encore développé. Or franchir les frontières au sein des institutions consiste à dépasser les contradictions inscrites au sein de ces 
mêmes institutions. Cela peut consister plus précisément à franchir les frontières entre différentes formes d'activité co-existant contradictoires (Engestrom, 2001) au sein même de cette organisation. Si les dirigeants des entreprises sont très focalisés sur l'hyper-rationalisation des processus de production en vue de l'augmentation des parts de marché pour qu'une entreprise se développe, nous émettons l'hypothèse qu'une organisation peut également se développer à travers le franchissement de frontières entre des systèmes de travail différents, voire qu'il existe une part en friche des organisations qui peut advenir à condition d'un franchissement de frontières intérieures à l'organisation. Ces franchissements de frontières peuvent donner lieu à diverses formes d'hybridation et de cristallisation. Pour Akkerman et Bakker (2011), l'hybridation se réfère à la combinaison d'ingrédients issus de différents mondes pour créer de la nouveauté qui peut prendre la forme de nouveaux outils, de nouveaux signes, de nouveaux concepts, ou encore de nouvelles pratiques et/ou de nouvelles collaborations. La cristallisation est quant à elle, la réification de l'expérience. Elle peut prendre forme à travers de nouvelles routines ou procédures permettant d'incarner ou rendre routinier ce qui a été créé ou appris. Les frontières et le franchissement de frontières sont des phénomènes malléables, puisque les frontières ne sont pas fixes une fois pour toutes, mais émergent d'une oscillation entre la fusion des mondes et la dissolution des frontières, et la reconstitution des frontières et l'imperméabilité des mondes. C'est un phénomène dynamique qui invite alors à se saisir du concept de frontière, non pas uniquement comme des barrières, mais comme des portes d'entrée heuristiques pour le chercheur-intervenant qui vise l'accompagnement d'une organisation en devenir.

5 La prise en compte des frontières au sein de l'analyse et de l'intervention invite les chercheurs à adopter des méthodologies écologiques (Hutchins, 1995; Suchman, 1987) ainsi que des unités d'analyse plus larges permettant d'appréhender au moins deux systèmes d'activité interconnectés. C'est clairement la voie proposée par Engeström (2001) et Akkerman \& Bakker (2011). Une seconde voie consiste à observer, examiner, comprendre les stratégies et les activités de réparation/reconstitution/réassemblage mises en œuvre par les personnes qui œuvrent aux frontières pour faire face aux fragmentations et aux discontinuités occasionnées par les frontières. Cette seconde voie est complémentaire de la première et vient éclairer l'organisation de manière intrinsèque à partir de l'activité mise en œuvre par les acteurs aux frontières.

\subsection{Développement des organisations et dimensions transitionnelles de l'activité humaine}

6 Que se passe-t-il aux frontières ? Comment observer, examiner et analyser la manière dont les personnes reconstituent des unités d'activités fragmentées par les frontières? Pour ne pas évincer ces questions, nous proposons de nous intéresser plus particulièrement à l'activité transitionnelle des personnes qui œuvrent aux frontières pour comprendre comment ces dernières tissent de la continuité pour elles-mêmes, et leur(s) collectif(s). Autrement dit, comment les collectifs créent aux frontières pour créer de la continuité intrinsèque et ne pas perdre leur dynamisme (Wenger, 1998). Nous allons dans un premier temps, préciser ce que nous entendons par approche transitionnelle. Nous commencerons par introduire les phénomènes transitionnels tels que définis par Winnicott, puis nous préciserons la manière dont cette approche nous permet de nous saisir des questions de continuité et de discontinuité. Nous prendrons 
pour cela l'exemple d'une première recherche empirique relative aux narrations de voyage qui est aux prémices de cette approche transitionnelle. Cela nous permettra également d'introduire la notion d'instruments transitionnels.

\subsubsection{Phénomènes transitionnels de Winnicott}

7 Dans Jeu et réalité, Winnicott (1971) propose une ontogenèse du jeu qui se situe dans un espace qu'il nomme tantôt «espace potentiel», tantôt "aire intermédiaire d'expérience ». Il introduit son propos en pointant l'absence de conceptualisation dans le champ psychanalytique d'une aire qui mériterait pourtant de l'attention et qu'il situe entre deux réalités : la réalité personnelle, intérieure et la réalité extérieure, partagée. Il débute la description des phénomènes transitionnels dans la petite enfance, matériau collecté grâce à sa pratique pédiatrique pour ensuite s'en détacher pour effectuer une proposition d'ordre plus général puisqu'il affirme que l'aire intermédiaire est universelle et perdure tout au long de la vie de l'individu (notamment dans les champs de la culture, de l'art, de la religion, de la création scientifique...). Ainsi le bout de couverture communément appelé doudou ou l'ours en peluche appartenant à l'enfant est conceptualisé par Winnicott comme un objet transitionnel, permettant à l'enfant de créer, imaginer, inventer et supporter l'absence de la mère via un prolongement symbolique. La présence de l'objet transitionnel aide l'enfant à tisser de la continuité en l'absence de sa mère : grâce à l'objet transitionnel, sa mère n'est pas totalement absente, puisque l'enfant est capable d'en conserver certains éléments auprès de lui. Cependant, l'enfant sait que l'objet transitionnel (couverture, peluche) ne remplace pas totalement sa mère, l'objet transitionnel est un relais lui permettant de sentir que l'expérience ne s'est pas abruptement terminée. Il permet le cheminement de l'enfant du subjectif vers l'objectif. Il marque la progression de l'enfant vers l'expérience vécue. Winnicott précise que ce n'est pas l'objet qui est transitionnel, mais que cet objet représente la transition du petit enfant qui passe de l'état d'union avec la mère à l'état où il est en relation avec elle, en tant que quelque chose d'extérieur et séparé. Ainsi Winnicott rappelle que ce qui est essentiel dans l'objet transitionnel, c'est la continuité pour le sujet.

8 Cet aperçu permet de comprendre que la matérialité n'est pas absente de ces phénomènes transitionnels. Ainsi, l'aire intermédiaire d'expérience a une fonction clairement identifiée par Winnicott qui consiste à maintenir reliée et séparée réalité intérieure et extérieure.

« Dans la vie de tout être humain, il existe la réalité intérieure, la réalité extérieure et une troisième partie c'est l'aire intermédiaire d'expérience, à laquelle contribuent simultanément la vie intérieure et la vie extérieure. Cette aire existe en tant que lieu de repos pour l'individu engagé dans cette tâche humaine interminable qui consiste à maintenir, à la fois séparées et reliées l'une à l'autre, réalité intérieure et réalité extérieure. L'aire intermédiaire se situe entre le subjectif et ce qui est objectivement perçu. »

9 Mais comment cette aire intermédiaire d'expérience permet de maintenir reliée et séparée réalité intérieure et extérieure ? Bien que Winnicott ne répertorie pas toutes les activités qui se déroulent dans cette aire intermédiaire d'expérience, il en évoque certaines telles que le rêve, le jeu, le travail thérapeutique qui serait une activité de jeu parmi d'autres. Ainsi, au fil de la lecture de Jeu et réalité, on comprend que cette aire intermédiaire d'expérience surgit dans l'entrelacs des mondes : au départ entre le bébé et la mère, ensuite entre l'enfant qui devient adolescent et la famille, progressivement 
entre l'individu et la société, au long cours entre la réalité intérieure et la réalité extérieure. Via des objets transitionnels remodelés dans cet entre-deux, le sujet peut manipuler les multiples facettes de sa réalité interne comme externe. Ce processus amène le sujet à jouer souplement de ses capacités à se dédoubler, à réparer, à relier des mondes ou des systèmes d'activités distincts.

\subsection{2. À l'origine de la perspective transitionnelle : récits de voyage \& instruments transitionnels}

10 La perspective transitionnelle trouve son origine dans une recherche au sein du domaine d'activité des narrations de voyage que nous avons eu l'occasion d'étudier au long cours (Bationo-Tillon, 2006, Bationo-Tillon, Folcher, \& Rabardel, 2010). Comme le rappelle Nicolas Bouvier, " on croit qu'on va faire un voyage, mais bientôt c'est le voyage qui vous fait et vous défait" (Bouvier, 1992). Le voyage correspond typiquement à des épisodes de vie où l'expérience tient à la manière dont le sujet affronte l'épreuve de l'évènement: soit le sujet rabat l'évènement sur du déjà connu, alors l'expérience s'installe comme enfermante ; soit le sujet se confronte à la nouveauté de l'évènement alors surgit une aire intermédiaire d'expérience qui ouvre le champ des possibles. Autrement dit, l'expérience constituée implique une clôture tandis que l'expérience ouverte implique une dynamique et l'émergence de phénomènes transitionnels (Winnicott, 1971). Cette distinction entre expérience ouverte et fermée (Pastré, 2011) n'est pas sans rappeler la distinction dessinée par Christin (2000) entre l'attitude du voyageur de celle du touriste. Ainsi, le voyageur préfère le chemin à la destination, s'ouvre à la découverte, à l'imprévu, au vécu, à la rencontre, il adopte une attitude d'ouverture à soi et au monde, accepte d'être déstabilisé et de sortir des normes habituelles. Tandis que le touriste s'installe dans une clôture de l'expérience. L'écriture nomade (Christin, 2000) établit un lien entre l'ici et l'ailleurs, mais aussi entre le passé, le présent et le futur. L'écriture du voyage s'apparente donc à un épisode d'indétermination offrant au voyageur-narrateur la possibilité d'inventer d'autres formes de reliance au monde, à soi-même et aux autres. Épisode d'indétermination créant un trait d'union entre le voyageur actuel et le narrateur en devenir. Nous avons nommé ce genre d'épisodes, des épisodes transitionnels. Le prisme du transitionnel nous invite à formuler l'interrogation suivante : comment la narration d'un épisode transitionnel en train de se faire permet-elle de maintenir ensemble et séparée une multiplicité de réalités?

11 Lorsqu'on se penche sur la diversité des personnes qui sont amenées à voyager et à élaborer des récits très divers (des passionnés de voyage, des ethnologues, des journalistes...), malgré la diversité foisonnante (propriétés des récits, nature et durée des voyages, finalité des récits...) des formes d'invariances sont à l'œuvre à travers les fragments collectés, assemblés et élaborés au cours du voyage (Bationo-Tillon, 2006 ; Bationo-Tillon, Folcher, \& Rabardel, 2010). Le voyageur rentre, riche de ses expériences et de ces fragments hétérogènes collectés/élaborés dans des espaces-temps différents du voyage. Au retour du voyage, le voyageur va étaler les fragments épars et arpenter tous ces éléments pour redéployer un monde constitué de fragments expérientiels : condition nécessaire pour élaborer un récit du voyage. Les phénomènes transitionnels permettent au sujet de garder le lien avec son expérience tout en l'objectivant, la regardant comme extérieure à soi. Or, les matériaux de la narration sont constitués d'éléments hétérogènes collectés, fabriqués ou capturés lors du déplacement, ils 
permettent donc dans un premier temps de découper le flux de l'expérience, de l'organiser. Puis dans un second temps, ils jouent le rôle de médiateurs pour le narrateur qui revisite des moments passés, s'imprègne à nouveau de l'expérience vécue pour élaborer un récit. Ils marquent la progression vers l'expérience vécue, vers la distanciation de l'expérience vécue tout en étant indispensables pour la réimprégnation de cette même expérience vécue. Il s'agit donc de phénomènes transitionnels au sens de Winnicott. Raison pour laquelle nous avons identifié et regroupé tous ces fragments épars sous le terme d'«instruments transitionnels", comme des indices de la capacité du narrateur à relier des mondes, comme des indices des va-et-vient entre monde extraordinaire du voyage et monde ordinaire et familier du narrateur, comme des relais permettant de sentir que l'expérience ne s'est pas abruptement terminée.

12 «Un instrument est transitionnel s'il ne contient pas uniquement l'idée de médiation (déjà présente dans la définition de l'instrument), mais assure la fonction de lien, de transition entre une situation spatiotemporelle et une autre situation spatiotemporelle. Un instrument devient transitionnel à partir du moment où un sujet lui attribue un statut particulier d'aide-mémoire, de témoin, de dépositaire des traces d'une expérience vécue. Il est constitué, fabriqué, approprié par le sujet. Il est transitionnel, car le sujet le fait transiter d'une situation s1 (lieu, espace, moment) à une autre situation s2. Son rôle en s2 est de faire référence à la situation S1. Le sujet l'utilise dans diverses activités discontinues dans le temps, donc dans des activités différentes ancrées dans des situations différentes. Ils sont des instruments de distanciation de l'expérience au moment de leur constitution, et instrument d'imprégnation de l'expérience passée au moment de leur réutilisation pour élaborer un récit. » (Bationo Tillon et al., 2010)

13 Ainsi appréhender la genèse des instruments transitionnels nécessite d'élargir la maille d'observation pour intégrer comme préconisé par Engeström (2001), au moins deux systèmes d'activités interconnectés (par exemple, les activités narratives dans le monde extraordinaire du voyage et les activités narratives dans le monde familier du narrateur), mais aussi d'élargir la maille diachronique pour suivre au long cours les élaborations, les ébauches et transformations successives au cours du temps.

\subsection{3. Élargissement de la perspective transitionnelle à l'organisation : processus commercial \& instruments transitionnels coopératifs}

14 Si la situation de voyage peut paraitre très éloignée de la vie des organisations qui planifient et gèrent à grande échelle, elle a le mérite de décrire les stratégies mises en œuvre par des sujets engagés dans un épisode d'indétermination, dans des épisodes dont on ne connait l'issue à l'avance. Dès lors que l'on s'intéresse aux organisations en devenir, il s'agit d'adopter un prisme permettant de comprendre comment au sein même de ces organisations qui ne cessent de planifier et prévoir, des activités transitionnelles peuvent advenir dans les îlots d'indétermination aux frontières, aux marges de ces organisations. Comment, au sein même des organisations, les personnes tissent-elles de la continuité pour elles-mêmes et pour les autres, permettant en cela de franchir les frontières qui provoquent de la fragmentation et de la discontinuité ? Ce faisant, comment les personnes, dans le cadre de leurs activités transitionnelles ordinaires, fondent l'organisation et permettent son efficacité? 
Dans la continuité des instruments transitionnels mis en évidence dans la sphère du voyage (Bationo-Tillon et al., 2010), nous avons identifié des instruments transitionnels coopératifs (Poret, 2015) qui re-dessinent les frontières au sein des organisations. Nous avons montré (Motté \& Poret, 2018; Poret, 2015) comment les systèmes d'information (SI) actuels non seulement fragmentent l'activité des opérateurs, mais les empêchent de reconstituer la continuité nécessaire au bon déroulement de leurs activités. Ils bricolent alors des instruments transitionnels en détournant les fonctions du SI pour retisser des unités d'activités brisées. Par l'entremise des instruments transitionnels coopératifs, les opérateurs reconfigurent donc les frontières. Ils rendent certaines frontières plus perméables au service de l'efficience d'une activité collective transverse (Motté \& Haradji, 2010). Nous allons montrer que fil du transitionnel est donc également mobilisé au sein des organisations, par l'entremise d'un second terrain relatif au processus commercial de relation clientèle, dans le cadre du traitement d'une demande de mise en service "bi-énergie» (Poret, 2015; Poret, Folcher, Motté, \& Haradji, 2016).

\section{Une nécessaire hybridation conceptuelle pour approcher les instruments transitionnels}

16 Aborder la question de l'activité transitionnelle comme une activité en train d'advenir au monde, conciliant des systèmes de rationalité et d'activité pluriels, nous semble particulièrement heuristique. Cette perspective nous invite à penser d'une part la gestation de l'activité dans la lignée des tenants de l'énaction (Theureau, 2004; Maturana \& Varela, 1980) afin de ne pas occulter la manière dont les activités émergent au monde. D'autre part, cette perspective nous a amenés à mobiliser la notion d'instrument transitionnel au sein de deux études qui se sont déroulées dans des domaines d'activité très différents, mais dont la confrontation nous semble fertile pour penser les organisations en devenir. Plus précisément, afin d'exemplifier la mise en œuvre de l'hybridation conceptuelle nécessaire à la perspective transitionnelle, nous allons détailler les deux recherches évoquées, à savoir celle relative au voyage, et celle relative au processus commercial de relation clientèle.

\subsection{Hybridation de l'approche instrumentale et du cours d'action}

17 Pour mettre en évidence des instruments transitionnels, il nous a été nécessaire d'accommoder notre outillage conceptuel et méthodologique de recueil et d'analyse des données. Les instruments transitionnels nous aident à mieux comprendre ce qui s'apparente à des ruptures d'un point de vue extrinsèque et qui pourtant révèlent paradoxalement des continuités plus profondes, plus intimes d'un point de vue intrinsèque. Pour rendre compte $d u$ cheminement diachronique des activités discontinues, le cours d'action (Theureau, 2004) et l'approche instrumentale (Rabardel, 1995) ont été mobilisés de façon complémentaire. Ces deux approches nous permettent d'effectuer des allers-retours entre une description et compréhension singulière et générique d'activités discontinues, mais également d'explorer leurs différentes temporalités (mailles synchronique et diachronique).

18 Ceci nous a amenés à explorer d'autres manières d'approcher la diachronie dans la mise en œuvre de l'approche instrumentale puisqu'il s'agit d'étudier des activités différentes 
dans des situations différentes. L'unité d'analyse classiquement proposée par l'approche instrumentale est la situation d'activité instrumentée. L'objet de l'activité relativement stable au cours du temps rend compte de la dimension finalisée de l'activité située. En effet, la notion de situation est centrale et Rabardel (2001) précise bien que toute activité instrumentée est toujours située et finalisée. Ainsi le modèle quadripolaire (cf. Figure 2) qui constitue le cœur de l'approche instrumentale se rattache toujours à une situation et à un objet de l'activité relativement stable. Ce cadre invite donc à découper le réel en identifiant les pôles et la structure de l'activité (sujetsinstrument-objet de l'activité).

Figure 1 : Modèle quadripolaire des situations d'activité instrumentée. Figure 1: Quadripolar model of situations of instrumented activity

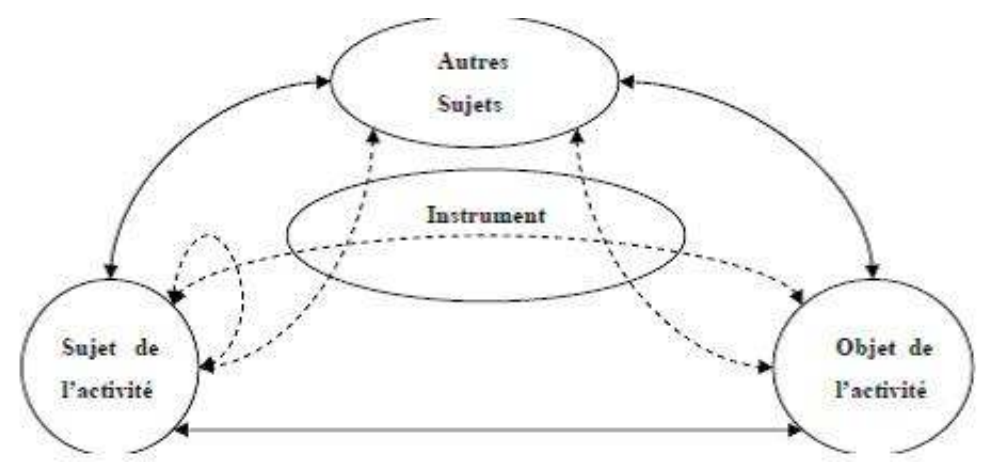

19 Ainsi l'approche instrumentale à travers ses concepts nous permet de rendre compte des continuités structurelles et fonctionnelles à travers l'organisation invariante de l'activité orientée vers un objet de l'activité. Il s'agit donc de trouver d'autres voies que la stabilité de l'objet de l'activité pour mettre en œuvre l'approche instrumentale de manière diachronique.

Pour explorer cette voie alternative, nous avons recours au cours d'action puisqu'en documentant précisément le point de vue de l'acteur à chaque instant par l'intermédiaire d'une phénoménologie de l'activité humaine, le cours d'action (Theureau, 2004) permet de décrire la construction de l'expérience. Par ailleurs, le cours d'action (Theureau, 2004, 2006) est un cadre théorique heuristique pour approcher la volatilité et les temps successifs des activités discontinues. Il offre la possibilité de maintenir les différentes histoires concomitantes aux activités dans lesquelles le sujet est engagé, et ceci est possible uniquement en restant au plus près du cours d'expérience singulier.

21 Cependant, l'optique de documenter les invariants de l'activité narrative dans sa diachronie nous amène à ne pas suivre la méthodologie du cours d'action de manière stricte puisque nous suivons la méthodologie d'élaboration du récit réduit sans explorer plus avant les possibilités offertes par la décomposition des signes hexadiques.

Ce sont ces hybridations conceptuelles ainsi que ces élaborations méthodologiques nécessaires à la mise en évidence des instruments transitionnels que nous décrirons plus en détail au sein de chacune des études. 


\subsection{Une hybridation mise en œuvre via une succession d'étapes}

Nous présentons là une succession d'étapes mise en œuvre sur les deux terrains présentés plus en détail dans la suite de cet article. Il s'agit du résultat d'une comparaison entre les méthodologies mises en œuvre sur chacun des terrains pour approcher des activités transitionnelles. Nous spécifierons davantage chacune de ces étapes pour chaque terrain, dans les parties correspondantes.

Dans un premier temps, nous avons eu recours à l'enregistrement audio-vidéo des activités suivi d'entretiens d'autoconfrontation (Theureau, 2004). Nous avons également recueilli les traces de ces activités (carnets, fiches, journées de bords, récits, inscriptions dans le système d'information).

Dans un second temps, une fois les matériaux empiriques recueillis, nous avons codé puis analysé ces données en quatre étapes ${ }^{2}$

1. Élaboration de récits réduits : nous avons utilisé la grille de lecture du cours d'action dans un premier temps afin de décrire le déroulement temporel de l'activité narrative selon des unités significatives pour l'acteur. Le cours d'action (Theureau, 2004, 2006) permet de retravailler, redécouper le temps chronologique pour constituer des unités significatives élémentaires (U.S.E) de l'acteur. Ces U.S.E.s correspondent aux actions, interprétations, focalisations, émotions ou communications de l'acteur. Elles émergent grâce à la description qui articule le point de vue de l'observateur chercheur (description extrinsèque) et le point de vue propre de l'acteur (description intrinsèque). Le récit réduit est constitué par l'enchainement chronologique des uses.

2. Constitution de classes de situations : Dans un second temps, nous avons mobilisé l'approche instrumentale pour segmenter ce déroulement des activités (autrement dit les récits réduits) en effectuant des extractions des situations d'activité instrumentée dans lesquelles nous avons ensuite identifié des schèmes et des instruments.

3. Compilation diachronique: Puis dans un troisième temps, nous avons cumulé les couches d'activité instrumentées de différentes temporalités T0/T1/T2... Tn. Cette étape de cumul était nécessaire pour appréhender la manière dont les activités instrumentées se sont sédimentées au cours du temps. Nous cherchions à comprendre comment les traces de l'activité se sont réifiées à différents moments dans le temps, autrement dit, comment elles se sédimentent et se transforment, successivement dans le cumul des couches temporelles au sein de systèmes d'activités hétérogènes.

4. Identification d'instruments transitionnels: Pour cette quatrième opération, nous nous appuyons sur la réversibilité rendue possible par l'analyse a posteriori, puisque nous partons de la forme aboutie de la trace de l'activité instrumentée (par exemple un fragment de récit final) pour remonter de manière archéologique dans les versions successives des traces de l'activité instrumentée pour comprendre ce qui s'est joué. Nous en déduisons les formes transitionnelles de l'activité par l'entremise de remodelages de la part du sujet qui non seulement transforme des traces (dimension visible), mais aussi et surtout son expérience (dimension invisible).

\section{Une hybridation conceptuelle qui permet de franchir différents niveaux de frontières}

Nous allons dans la partie empirique de cet article, donner à voir à travers deux recherches hétérogènes du point de vue de leurs temporalités, de leurs domaines d'activités, de leurs finalités et de la granularité d'analyse, la manière dont des 
instruments transitionnels ont été mis en évidence grâce à l'hybridation de l'approche instrumentale et du cours d'action.

\section{1. Écrire le voyage}

SI l'écriture du voyage surgit chez un sujet engagé dans l'entrelacs des mondes, elle ne peut que contribuer à la percolation de ces mondes. Mais comment ces mondes se mêlent-ils, s'enchevêtrent-ils concrètement ? Qu'est-ce qui émerge de cette percolation des mondes? C'est ce que nous allons découvrir en nous appuyant sur les traces matérielles des activités narratives, comme des indices, des témoins de ce qui se joue. Le prisme du transitionnel nous invite à formuler une série d'interrogations : quelle est la nature de l'engagement du voyageur-narrateur? Comment la narration du voyage en train de se faire permet-elle de maintenir ensemble et séparée une multiplicité de réalités ? Précisons d'emblée que nous adoptons une acception élargie de l'écriture. En accord avec Bruner (1990), nous pensons que la narration est une forme d'expression, un mode de pensée qui permet à l'homme de construire des interprétations du monde, ainsi que de donner un sens à ses expériences pour les partager avec les autres. Ici, nous délimitons les activités narratives aux récits d'évènements vécus et nous incluons tous les artefacts (photographie, vidéo, dessin, écrit...) pouvant s'articuler au cours de la production d'un récit. Nous nous intéressons tout autant aux activités de recueil au cours du voyage (prise photographique, prise de notes...) qu'aux activités de mise en forme, cohérence des éléments recueillis après le voyage. Autrement dit, nous nous intéressons à la narration en train de se faire, à l'émergence du sens de l'expérience en train de se faire auprès d'un panel varié de narrateurs-voyageurs (2 expéditeurs polaires, et 10 autres personnes aux profils divers (journalistes, ethnologues, blogueurs). Des enregistrements vidéo ont été réalisés ${ }^{3}$, d'une part pour documenter l'activité narrative dans son aspect temporel et dynamique, d'autre part pour que le chercheur ne soit pas en présence des sujets, ni même observateur des activités en temps réel. Des entretiens en resitu subjectifs (Rix \& Biache, 2004) et entretiens d'autoconfrontation à partir des traces de l'activité ont été menés a posteriori. Ces entretiens ont été réalisés selon une démarche commune de questionnement, en insistant sur les questions autour des préoccupations, des éléments auxquels le sujet portait attention, ainsi que sur l'explicitation des actions effectuées (Theureau, 2004 ; Vermersch, 1994).

\subsection{1. Élaboration de récits réduits}

Comme le montre le tableau ci-dessous, nous avons mobilisé dans un premier temps la démarche conceptuelle et méthodologique du cours d'action. Après avoir transcrit l'intégralité des entretiens d'autoconfrontation, et codé l'ensemble des enregistrements vidéo, il s'agit de constituer des récits réduits au sens de Theureau (2004) en fusionnant deux flux de codage que nous mettons en correspondance : à savoir une description objectivable/extrinsèque et chronologique de l'activité ( 5 premières colonnes du tableau ci-dessous) avec une description intrinsèque de ce qui se passe pour l'acteur, pas à pas, issue de la transcription de l'autoconfrontation ( $6^{\text {ème }}$ colonne du tableau 1 cidessous). 
Tableau 1 : Codage et transcription.

Table 1: Coding and transcription

\begin{tabular}{|c|c|c|c|c|}
\hline temps & $\begin{array}{l}\text { Espace } \\
\text { de travail }\end{array}$ & Action du sujet & \begin{tabular}{|l|} 
Environ- \\
pement
\end{tabular} & \begin{tabular}{|l|} 
Contean de ce \\
qui est earegistré
\end{tabular} \\
\hline$b^{00}: 04: 1$ & & $\begin{array}{l}\text { P1 regarde } \\
\text { montre et enonce } \\
\text { 'sititude à P2 }\end{array}$ & \begin{tabular}{|l|}
$\mathrm{P} 2$ \\
demande \\
'altitude \\
31 P1 \\
1
\end{tabular} & \\
\hline $\begin{array}{l}00: 05: 0 \\
3\end{array}$ & & $\begin{array}{l}\text { Avance son } \\
\text { stylo pres du cahier }\end{array}$ & & \\
\hline $0^{00: 05: 1}$ & anoto & $\begin{array}{l}\text { commence is écrire } \\
\text { sur la page de } \\
\text { ganche } \\
\text { et il souligne le } \\
\text { texte }\end{array}$ & & \begin{tabular}{|l|} 
bilan \\
programme \\
LME"
\end{tabular} \\
\hline $\begin{array}{l}00: 05: 2 \\
8\end{array}$ & amoto & $\begin{array}{l}\text { If va à la ligue ex } \\
\text { ecrit }\end{array}$ & & $\begin{array}{l}\text { - cadrage des } \\
\text { problématiques." } \\
\text { problemmatiques } \\
\text { générales et } \\
\text { problématiques } \\
\text { singulières }\end{array}$ \\
\hline
\end{tabular}

00:04:00 Comme P2 a acheté cette montre là, on essaie de savoir comment elle fonctionne. C'est la montre d'expéditeur, je régle (altimètre, Thermomètre, heure, boussole) .... Donc la on essaie toujours de voir comment marche cette boussole électronique. $00: 05: 00 \mathrm{~A}$ ce moment la tu conemences a dcrire? Attends j'ai pas encore démarré. Donc là jhésite (je démarre à gauche ou à droite) parce que je n'aime pas commencer à gauche comme cela.

Finalement th demarres a ganche, et th dcris bilan du programme $L M E$ ?

oui en fait comme c'est pas de la chrono, je peux le mettre sur la page de gauche et easuite je commencerai la chrono sur la page de droite; voila j'y ai pensé dans la joumée, et je veux clanifier, c'est important toujours de mettre noir sur blanc.

00 :06:00 The éris sous forme de liste?

Ou il y a quelques points à faire auxquels je veux faire attention. Heu... donc là c'est cadrage entre les problématiques générales et singuliéres des acteurs, trouver une espèce d'équilibre, après un problème

La fusion de ces éléments nous a permis de constituer des récits réduits constitués d'unités significatives élémentaires. Nous n'avons pas décomposé chacune de ces unités en signe tétradique ou hexadique ${ }^{4}$ (Theureau, 2004), cependant, nous avons documenté pour chaque U.S.E, l'objet du signe tétradique ${ }^{5}$ (la préoccupation pour l'acteur).

Tableau 2 : Récits réduits et objets.

Table 2: Reduced narrative and objects

Image

100605680000455600001AE2779667CF0B1EBBE0.emf

\begin{tabular}{|c|c|c|}
\hline temps & Déroulement des USES & objets \\
\hline $00: 05: 03$ & $\begin{array}{l}\text { 7-regarde anoto en hésitant à commencer à droite ou } \\
\text { à gauche }\end{array}$ & \multirow[t]{8}{*}{$\begin{array}{l}\text { Garder trace de réflexions } \\
\text { (choses à faire) pragmatiques }\end{array}$} \\
\hline $00: 05: 11$ & $\begin{array}{l}\text { 8-écrit en nommant la liste future (sur la page de } \\
\text { gauche) bilan du programme LME et souligne }\end{array}$ & \\
\hline \multirow[t]{2}{*}{$00: 05: 26$} & 9-écrit en dessinant un point & \\
\hline & 10-écrit en énumèrant une première idée & \\
\hline \multirow[t]{2}{*}{$00: 05: 58$} & 11-écrit en dessinant un point & \\
\hline & 12-écrit en énumèrant une deuxième idée & \\
\hline \multirow[t]{2}{*}{$00: 06: 03$} & 13-écrit en dessinant un point & \\
\hline & 14-écrit en énumérant une troisième idée & \\
\hline $00: 06: 20$ & 15-lève la tête & \multirow{2}{*}{$\begin{array}{l}\text { (autre) Interagir avec son } \\
\text { environnement }\end{array}$} \\
\hline $00: 06: 22$ & $\begin{array}{l}\text { 16-discute avec } \mathrm{P} \text { et } \mathrm{G} \text { en leur parlant dine carte à } \\
\text { envoyer aux membres LME }\end{array}$ & \\
\hline
\end{tabular}

30 Ainsi le cours d'action nous permet de détacher le fil d'activité qui nous intéresse de l'environnement dans lequel elle se déroule sans en occulter la dimension située puisque l'activité est appréhendée dans ce cadre conceptuel comme émergente dans l'interaction du sujet et de l'environnement pas à pas. En ayant recours à cette élaboration des récits réduits, nous avons mis en œuvre l'approche phénoménologique du cours d'action pour dés-intriquer les activités narratives des autres activités avec lesquelles elles sont enchevêtrées au cours du voyage notamment.

\subsubsection{Constitution de classes de situation}

31 Dans un second temps, nous menons une opération qui consiste à découper les récits réduits pour les traduire en classes de situation. Étant donné la volatilité des activités narratives, nous prenons appui sur les objets du signe triadique/préoccupation du cours d'action pour découper les récits réduits et constituer des classes d'activité 
narrative instrumentée. Alors que dans les théories de l'activité, l'objet de l'activité ne change pas de moment à moment, dans notre contexte, le cours d'action nous permet d'appréhender les objets émergeant pas à pas. C'est donc l'analyse a posteriori qui nous permet de décider de regrouper toutes les U.S.E rattachées au même objet, en classes de situation, pour identifier ensuite au sein de ces classes les schèmes et les instruments. Ainsi, si l'on s'appuie sur le tableau 2: une classe de situation instrumentée a pu émerger autour de: "garder des traces de réflexion pragmatiques». Cette classe de situation a permis de regrouper l'ensemble des séries ou séquences rattachées à cet objet, pour en extraire dans un second temps des invariants (schème et/ou instruments) (pour plus de détails, voir Bationo-Tillon, 2006).

Poursuivons, par la troisième étape qui nous permet d'intégrer la diachronie dans l'analyse comme le montre le schéma ci-dessous autour de l'étude menée auprès de deux expéditeurs polaires :

Figure 2 : analyse synchronique et diachronique.

Figure 2: synchronic and diachronic analysis

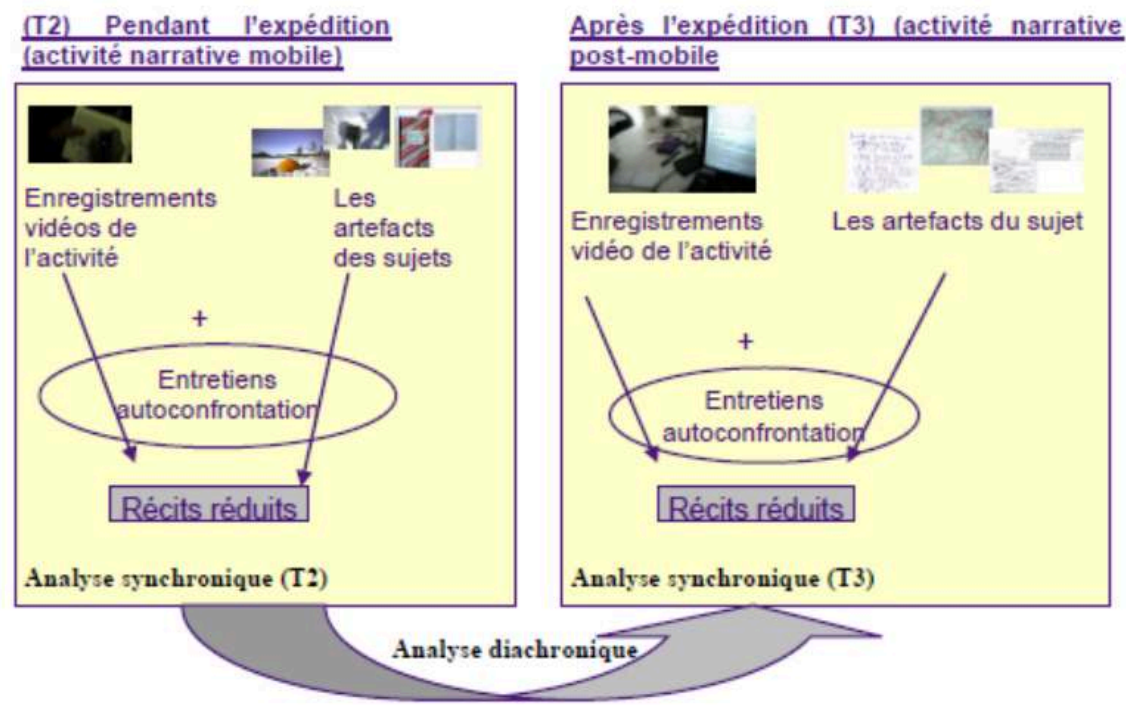

\subsubsection{Compilation diachronique}

$\mathrm{Au}$ cours de cette étape, le chercheur intervient puisqu'il compile des moments d'activité pour accéder à la maille diachronique (T2/T3), en associant des épisodes d'activité qui n'ont pas le même objet de l'activité, mais qui, en revanche, sont des épisodes d'activité présentant une continuité du point de vue de la matérialité des traces. Ce sont bien les traces élaborées à T2, qui sont reprises et transformées à T3. Il s'agit donc plus d'une continuité sémiologique (continuité du représentamen ${ }^{6}$ ) et matérielle que d'une continuité d'objet d'activité, puisque l'objet de l'activité se transforme en chemin. L'approche instrumentale nous permet donc de décrire les différences de statut des fragments recueillis à T2 et T3, tandis que le cours d'action nous permet de percevoir les continuités-discontinuités sémiologiques à l'œuvre. Ainsi, les fragments recueillis sont des indices de ce qui fait sens pour le sujet, encapsulent le sens pour le sujet à un moment donné : dans un autre contexte, les sujets remobilisent et ré-agencent ces éléments recueillis dans des ensembles plus grands (une forme de 
récit particulière : par exemple le journal de bord de l'expédition à destination de la communauté des expéditeurs polaires), ils recomposent et reconfigurent le sens de leur expérience, d'une manière parfois très différente de ce qu'ils feraient pour l'élaboration d'un article scientifique par exemple. Ainsi, la maille diachronique est dessinée par le chercheur - intervenant qui choisit de se focaliser sur l'élaboration d'un des récits du narrateur. Se faisant, il cumule l'activité narrative des sujets à différents moments/ endroits du voyage, il documente la manière dont le narrateur franchit les frontières des mondes, en bricolant de la continuité pour lui-même tout en élaborant un récit spécifique comme le montre l'illustration ci-dessous :

Tableau 3 : La diversité des traces de l'activité narratives dans des temps successifs. Table 3: The diversity of traces of narrative activity at successive points in time

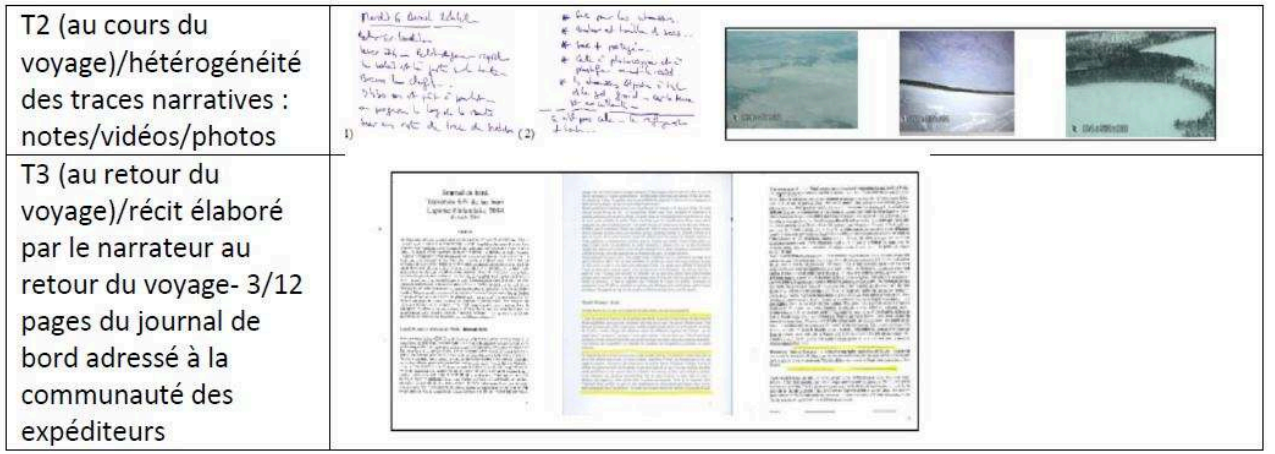

34 Ainsi le chercheur-intervenant, a cumulé a posteriori tous les moments et toutes les traces narratives ayant concouru à l'élaboration du journal de bord adressé à la communauté des expéditeurs. C'est le cumul de ces activités hétérogènes guidé par les traces de l'activité narrative concourant à élaborer ce document finalisé à l'adresse de la communauté des expéditeurs qui permet d'introduire de la diachronie dans l'analyse.

\subsubsection{Instruments transitionnels}

Dans cette quatrième étape, le chercheur va donc identifier au sein des activités cumulées, les reprises sémiologiques et matérielles du narrateur. Il s'agit donc bien dans cette étape ultime de l'analyse de combiner les mailles du cours d'action et de l'approche instrumentale, en combinant les reprises matérielles (donc en examinant la transformation matérielle des matériaux de la narration) et sémiologiques (la transformation du sens dans un autre contexte/la manière dont se tisse, se transforme du sens nouveau ou encore la manière dont se tisse de la continuité de sens pour le sujet). Dans cette étape, il s'agit donc pour le chercheur d'étaler les traces cumulées (écrits, notes, fragments, photos, etc.) de l'activité narrative instrumentée en respectant le décours temporel afin de considérer ces traces comme des indices qui de ce qui émerge dans entrelacs de mondes, comme des indices du remodelage de l'expérience des sujets à l'œuvre au sein des activités narratives.

Ceci nous permet à ce stade d'apercevoir les diverses formes d'activités narratives diachroniques révélées par les instruments transitionnels à savoir des activités linéaires qui tendent à reprendre à l'identique un contenu comme l'illustre cet exemple d'instrument transitionnel miroir mis en évidence dans l'activité d'un des expéditeurs dans le tableau 4 : 
Tableau 4 : Instrument transitionnel miroir. Table 4: mirror transitional instrument

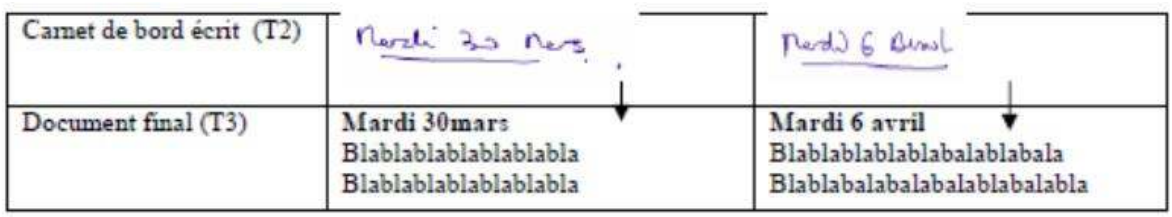
cités se sont généralisées au cours des dernières décennies. Si cette fragmentation peut être liée à des besoins en compétences spécifiques, elle peut également résulter du recentrage des entreprises sur leur « cœur de métier », les conduisant à externaliser les autres activités au sein d'organisations en réseau de type "supply chains". À l'heure actuelle, au sein de ces formes organisationnelles, coopérer est un enjeu central. La coopération au sein des processus et des supply chains est recherchée, notamment par l'intermédiaire du "Supply Chain Management" dont un des objectifs principaux réside dans l'«intégration", notion employée pour décrire la nature plus coopérative et l'intensité des relations dans la supply chain (Fawcett \& Magnan, 2002). Les relations contractuelles clients-fournisseurs se veulent également plus collaboratives (Brulhart \& Favoreu, 2006). Malgré cette injonction à la coopération destinée à sortir des « silos » qui ont émergé de la fragmentation, à l'heure où la transversalité est prônée comme un 
enjeu essentiel au sein de toutes les organisations, force est de constater que le décloisonnement n'est pas complètement effectif (Poret et al., 2016).

La perspective transitionnelle nous permet de sortir de cette impasse, et de comprendre la coopération de manière intrinsèque, à partir de l'activité humaine. Dans la fragmentation apparente des activités au sein du processus commercial que nous avons étudié se développe une activité collective transverse (Motté \& Haradji, 2010; Poret, 2015), fondée par les acteurs du processus qui relient les étapes entre elles, maintiennent une continuité du traitement collectif de la demande du client malgré la discontinuité qui caractérise le processus. Par leurs activités transitionnelles, ils franchissent les frontières temporelles et spatiales du processus, permettant son efficacité, ainsi que la satisfaction du client.

Il s'agit alors d'appréhender la façon dont les acteurs tissent de la continuité, non pas entre eux-mêmes ici et maintenant et eux-mêmes plus tard, comme cela peut être le cas pour le narrateur de voyage, mais entre eux-mêmes ici et maintenant et d'autres acteurs plus tard : ceux qui seront chargés de reprendre le traitement de la demande du client ultérieurement. Contrairement au fil transitionnel qui peut être tiré pour les narrateurs de voyage, il y a donc là une différence importante: la discontinuité de l'expérience. L'acteur qui collecte des fragments épars à l'instant $t$ n'est pas celui qui sera amené à les ré-agencer; ces fragments ne sont pas des fragments expérientiels pour l'acteur qui est chargé de poursuivre le traitement de la demande du client.

Figure 3 : Les étapes constitutives du processus de traitement de la demande de mise en service bi-énergie.

Figure 3: The constituent stages of processing requests for dual-fuel connection

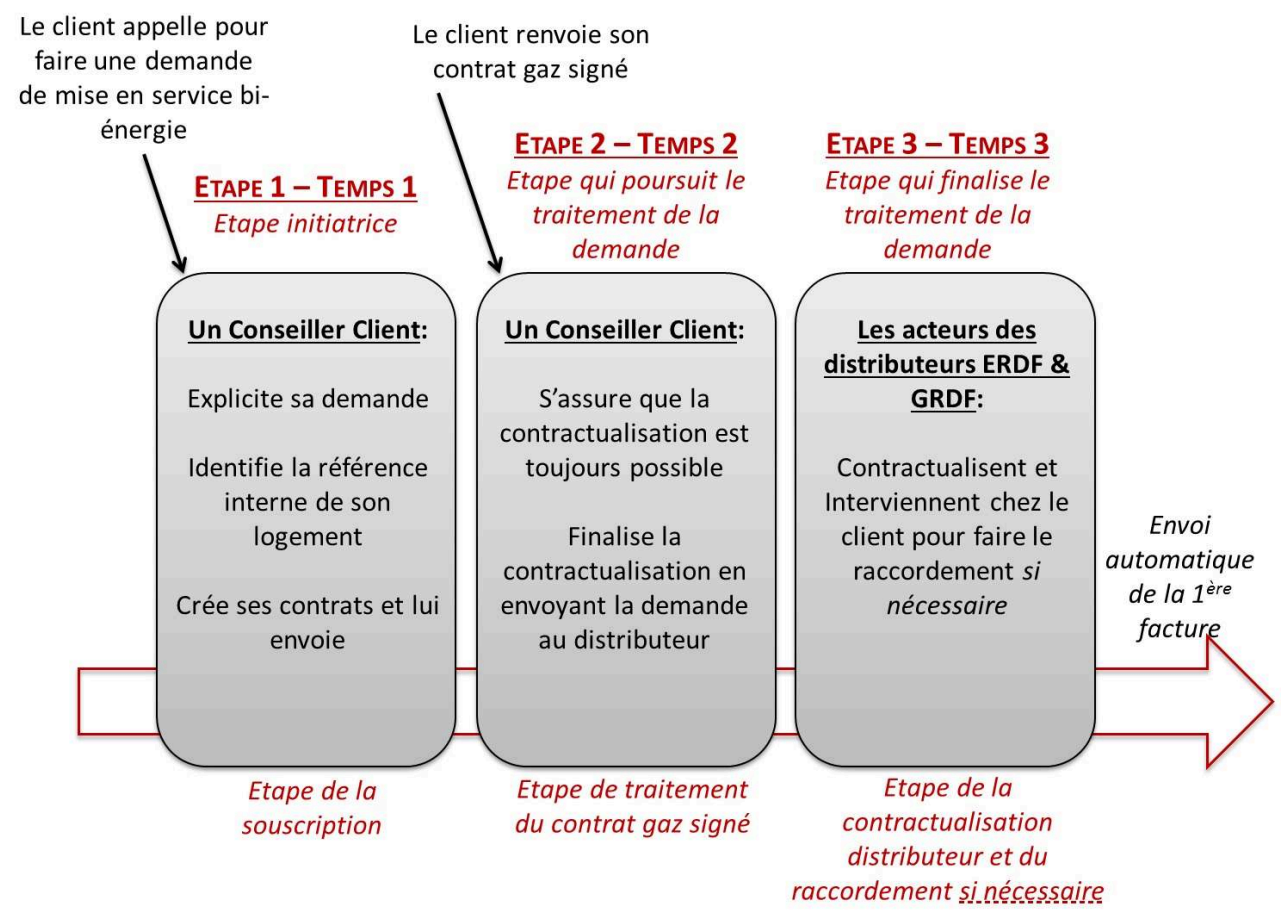

Pour que la demande du client soit traitée du début à la fin, ce processus comporte nécessairement plusieurs étapes (Figure 1), qui sont traitées par différents acteurs, dans et hors de l'organisation. Ce processus se caractérise par une distribution 
temporelle et spatiale des contributions de différents acteurs qui doivent, dans cette fragmentation, construire collectivement la réponse à la demande du client.

L'activité collective transverse au sein de ce processus prend la forme d'activités transitionnelles qui permettent de maintenir une continuité temporelle et d'assurer la progression du traitement de la demande du client. A chaque étape du processus en effet, les conseillers clients :

- Ont besoin de comprendre l'histoire du traitement de la demande jusqu'à cet instant, et pour cela ils s'engagent dans une enquête historique qui met en intrigue des traces qui ont été laissées par les acteurs qui ont précédé dans le traitement de cette demande ;

- Mettent en œuvre ce qui leur incombe au niveau de cette étape, pour que le traitement de la demande se poursuive ;

- Transmettent et/ou mettent en archives des éléments relatifs à l'étape qu'ils viennent de traiter. Ils laissent ces éléments dans le système d'information, à l'attention de ceux qui auront à prendre la suite du traitement de cette demande, ailleurs et plus tard.

Le mouvement général de l'articulation que nous avons proposée a consisté à partir d'une phénoménologie de l'activité humaine - par l'intermédiaire de la modélisation de l'histoire des transformations du système des ouverts ${ }^{7}$ à l'instant $t$ - et d'aller progressivement vers la mise en évidence des instruments transitionnels coopératifs. Le point charnière de cette articulation était constitué par le point de vue de l'acteur et par les notions qui portent ce point de vue dans chacune des approches.

\subsection{1. Élaboration de récits réduits concernant chaque étape du processus}

47 Notre recueil de données a consisté à réaliser des enregistrements audio-vidéo en continu de l'activité des conseillers-clients engagés dans le traitement des différentes étapes constitutives de la demande de mise en service bi-énergie. Nous étions assis à côté du conseiller-client, caméra centrée sur leur écran d'ordinateur et prenions des notes sur le déroulement de leur activité ainsi que sur les verbalisations du client qui était en double-écoute. Immédiatement après le traitement de l'étape par le conseillerclient nous revenions avec lui sur ce qu'il venait de faire, dans le cadre d'entretiens nommés "post-traitement du cas » qui avaient le même objectif que les entretiens d'autoconfrontation à savoir l'expression de la conscience préréflexive de l'acteur (Theureau, 2004, 2006). La spécificité de l'activité collective considérée dans le cadre de ce travail nous a amenés à assigner un second objectif à ces entretiens : revenir avec les acteurs sur l'avant et l'après de leur propre contribution afin de nous permettre de situer plus largement cette contribution dans la continuité du traitement de la demande. En tout, nous avons recueilli $95 \mathrm{~h}$ d'enregistrement audio-vidéo, auprès de 24 conseillers-clients et 2 de leurs responsables hiérarchiques directs. Nous détaillons maintenant plus précisément les différentes étapes de l'hybridation entre l'approche instrumentale et le cours d'action nous ayant permis de mettre en évidence les instruments transitionnels coopératifs.

Sur la base de "chroniques ", qui correspondent à une transcription de nos données sous forme de tableau à plusieurs colonnes détaillant le comportement observable et les actions informatiques des acteurs, les verbalisations simultanées et les verbalisations en autoconfrontation, nous avons construit des récits réduits. Nos récits réduits ont mis en évidence des séquences - continues et discontinues -, des macro- 
séquences, des séries et des synchrones, ainsi que l'ouvert qui fonde ces unités significatives à chaque instant (Tableau 5).

Tableau 5 : Identification des unités élémentaires significatives de rang plus élevé et de l'ouvert qui les fonde à chaque instant.

Table 5: Identification of significant elementary units of higher rank and of the opening that founds them at all times

\begin{tabular}{|c|c|c|c|c|}
\hline $\begin{array}{l}\text { Unités Significatives } \\
\text { Elémentaires (USE) }\end{array}$ & Séquences & Séries & Macro-Séquences & Ouvert \\
\hline 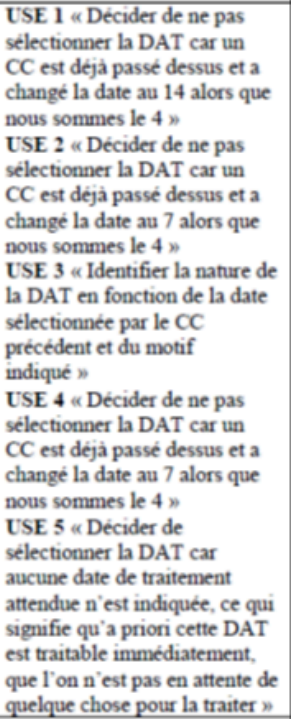 & $\begin{array}{c}\text { Trouver une DAT } \\
\text { qu'elle peut traiter } \\
\text { dès à présent }\end{array}$ & & $\begin{array}{c}\text { Trouver une DAT } \\
\text { traitable }\end{array}$ & $\begin{array}{c}\text { Traiter le cas } \\
\text { de DAT } \\
\text { relativement à } \\
\text { un } \\
\text { redressement } \\
\text { de facture }\end{array}$ \\
\hline $\begin{array}{l}\text { USE } 6 \text { « Comprendre qu'il } \\
\text { s'agit d'un client migré parce } \\
\text { que son numero client } \\
\text { commence par } 5000 \% \\
\text { USE } 7 \text { " Accéder au dossier } \\
\text { du client à partir d'un } \\
\text { copiet/coller de son numéro } \\
\text { client } \%\end{array}$ & $\begin{array}{l}\text { Accéder au dossier } \\
\text { du client dans } \\
\text { SNMM car il s'agit } \\
\text { bien d'un client } \\
\text { migré }\end{array}$ & & $\begin{array}{l}\text { Comprendre de } \\
\text { quoi il s'agit } \\
\text { exactement }\end{array}$ & \\
\hline
\end{tabular}

Nous avons réalisé des récits réduits pour plusieurs cas relatifs à chaque étape du traitement de la demande de mise en service bi-énergie. Nous disposions ainsi de récits réduits relativement au point de vue de conseillers clients traitant l'étape de souscription (Étape 1 - Temps 1), ainsi que de conseillers clients traitant l'étape de traitement du contrat gaz signé (Étape 2 - Temps 2), etc.

Enfin, chaque récit réduit a donné lieu à la construction d'un graphe d'analyse du cours d'action dont l'objectif était de rendre compte de la dynamique de construction du cours d'action des conseillers clients en mettant en évidence 1) les unités significatives du cours d'action de chacun des conseillers distinguées selon les ouverts qui les fondent à chaque instant et 2) l'agencement temporel des différents ouverts structurant l'engagement du conseiller client. La comparaison de l'ensemble de ces graphes nous a permis de monter en généralisation et d'identifier des ouverts et des structures significatives types, c'est à dire qui se retrouvent dans chacune de ces modélisations. Par exemple, l'ouvert " articuler son activité à celle des autres " se retrouve dans l'activité de chacun des conseillers-clients engagés dans le traitement d'une étape de la demande de mise en service bi-énergie. 


\subsubsection{De l'ouvert type à l'objet de l'activité, et de la structure significative type à la mise en évidence des instruments}

51 L'articulation avec l'approche instrumentale intervient à partir de cette étape. À partir de la connaissance des dimensions typiques de l'activité des conseillers clients, nous sommes passés : fondamentales de l'activité des acteurs et correspondent à un haut degré de généralisation. Le caractère typique de ces structures, au travers de la notion de typicalité, renvoie directement au concept de schème d'action instrumentée de l'approche instrumentale. En effet, le caractère typique des structures significatives contient deux dimensions présentes dans les schèmes, à savoir la régularité, l'invariance, la similarité appréhendée à un haut degré de généralisation de l'activité humaine. C'est la raison pour laquelle nous avons choisi de partir des séquences-types pour poser des hypothèses de schèmes d'action instrumentée. Ainsi par exemple, les séquences types "Transmettre des éléments utiles au traitement ultérieur de la demande » et «Laisser des éléments relatifs au traitement de l'étape qu'il vient de traiter " nous permettent de faire l'hypothèse de l'existence d'un schème relatif à la transmission d'éléments à destination de ceux qui interviendront ultérieurement dans le traitement de la demande.

Enfin, pour que la mise en évidence des instruments des conseillers clients soit complète nous avons examiné les ressources artefactuelles systématiquement mobilisées avec le ou les schème(s) identifié(s). 


\subsubsection{Compilation diachronique : du niveau de l'étape au niveau du processus de traitement de la demande dans son ensemble}

59 La troisième étape a alors consisté à prendre de la hauteur et à cumuler des couches d'activité instrumentée de différentes temporalités. Il s'est agi de passer du niveau de chacune des étapes pour remonter au niveau du traitement de la demande dans son ensemble. Ce changement de niveau s'est fondé sur la connaissance de l'activité des acteurs à chaque étape, et a été nécessaire afin de rendre visible la continuité qui résulte de ce que les conseillers clients mettent en œuvre à chaque étape pour articuler leurs contributions respectives. Nous avons ainsi relié la connaissance des instruments à chaque étape au niveau du traitement de la demande dans son ensemble pour faire émerger et accéder à la continuité portée par ces instruments. Sans ce cumul de couches d'activité instrumentée, réalisé par le chercheur intervenant, les activités transitionnelles et les instruments développés par les acteurs au service de ces activités seraient plus difficilement appréhendables.

En effet, comme cela a été mis en évidence dans l'étude relative au voyage, ces activités transitionnelles ne s'attrapent pas en suivant le fil des objets de l'activité. Par exemple, pour ce qui est de l'activité collective transverse au sein du processus commercial, nous avons ainsi identifié un ouvert récurrent dans l'activité des conseillers clients, "Articuler son activité à celle des autres acteurs du traitement de la demande », qui met en évidence la considération, par les conseillers clients, de l'existence d'un collectif engagé dans le traitement de la demande au-delà du seul traitement de l'étape qui leur incombe. Cet ouvert type, que nous avons considéré comme un objet d'activité, est orienté vers l'activité collective transverse. Mais l'examen des objets de l'activité n'est pas suffisant pour remonter le fil de l'activité collective transverse et de ses instruments. Pour rappel, cette activité collective est fondée par la mise en intrigue du matériau laissé par d'autres acteurs pour reconstruire l'histoire du traitement de la demande jusqu'à cet instant, et de la transmission/mise en archives d'éléments à destination de ceux qui suivront dans ce traitement. Si l'objet de l'activité relatif à la transmission/mise en archives est directement orienté vers le collectif, cela n'est pas le cas de l'objet de l'activité relatif à la mise en intrigue du matériau qui a été laissé par les autres. Lorsqu'il laisse des éléments à destination d'autrui, le conseiller client articule volontairement sa contribution avec celle d'autrui qui suit dans le traitement de la demande, en laissant des éléments qui ne sont plus directement pertinents pour lui, mais pour celui qui devra prendre le relai ailleurs et plus tard. Cela n'est pas le cas lorsqu'il prend le relai dans le traitement de la demande. Lorsqu'il met en intrigue les traces laissées par les autres, le conseiller client est engagé dans le traitement de l'étape qui lui incombe et n'a pas conscience qu'il est en train d'articuler sa contribution avec ceux qui ont précédé. Il s'est alors agi, pour le chercheur intervenant, d'examiner la provenance du matériau qui est mis en intrigue lorsque les conseillers clients prennent le relai dans le traitement d'une demande. C'est bien le fil de la « trace » investigué par le chercheur intervenant qui permet d'accéder aux dimensions transitionnelles de l'activité, sans quoi il risquerait de passer à côté. 


\subsubsection{Les instruments transitionnels coopératifs, instruments de la continuité dans les organisations}

61 Les instruments de la mise en intrigue et de la transmission/mise en archives sont des instruments transitionnels coopératifs au service de l'articulation Passé-Présent-Futur à chaque étape, car ils permettent à l'acteur traitant l'étape actuelle de la demande à la fois de reconstruire des dimensions passées par le moyen de la mise en intrigue, et d'anticiper le futur de ce traitement par le moyen de la transmission/mise en archives. En cela ils permettent la présentification du passé et la présentification du futur du traitement d'une demande à chaque acteur engagé dans le traitement d'une étape de cette demande. Si on quitte ce point de vue par étape pour élargir la focale et adopter le plan général du traitement de la demande, ces instruments permettent une transition entre le futur du traitement de la demande envisagé par l'acteur 1 à $t$, et le passé de ce traitement envisagé par l'acteur 2 à $t+1$ (Figure 4). En cela, ils constituent les instruments de la continuité temporelle au cœur des organisations.

Figure 4 : Les Instruments Transitionnels Coopératifs, instruments de la continuité au cœur des organisations.

Figure 4: Cooperative Transitional Instruments, instruments of temporal continuity at the heart of organisations

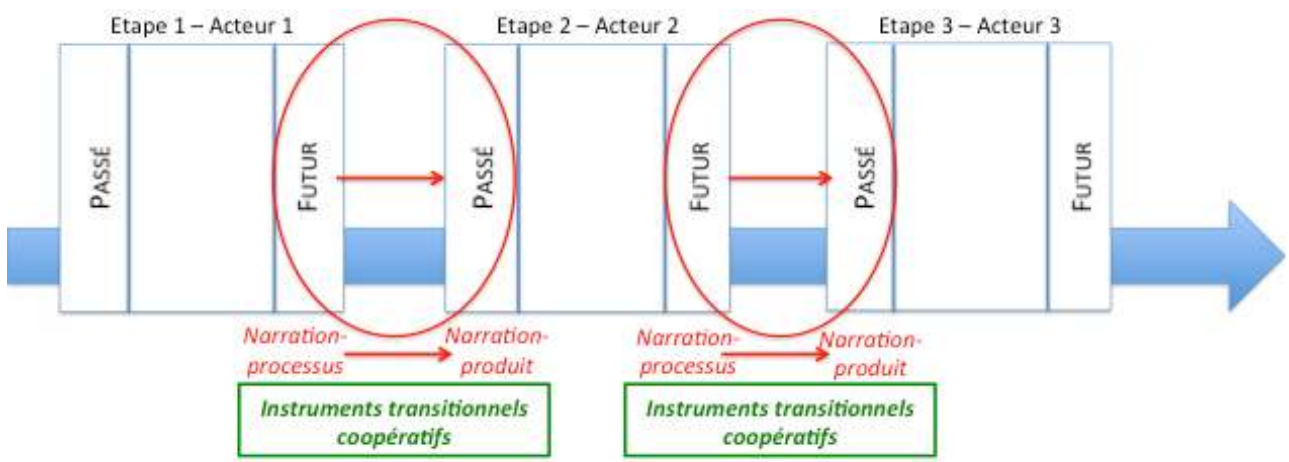

En effet, ils permettent la transition entre une étape 1 à $t$ et une étape 2 à $t+1$, en maintenant une certaine continuité. Cette continuité, à l'inverse des instruments transitionnels mis en évidence par Bationo-Tillon (2006) relativement à l'activité individuelle, ne peut pas être une continuité de l'expérience puisque l'acteur qui agit au niveau de l'étape 1 à $t$ et celui qui agit au niveau de l'étape 2 à $t+1$ ne sont pas les mêmes. Autrement dit, ces instruments permettent de maintenir une certaine continuité dans le traitement de la demande, malgré une discontinuité de l'expérience entre chacune des étapes. Cette discontinuité de l'expérience entre les différentes étapes est à l'origine du fait que les acteurs ne peuvent pas faire appel à leur mémoire personnelle. C'est la raison pour laquelle, à l'instar des historiens, ils mettent en œuvre une activité d'enquête historique dont l'objectif est de reconstruire des dimensions passées du traitement de la demande.

Enfin, le caractère plurivoque et pluri-adressé des instruments transitionnels des narrateurs de voyage se retrouve dans les instruments transitionnels coopératifs des conseillers clients. En effet, notre recherche a distingué la transmission et la mise en archives lorsqu'il s'agissait, pour les conseillers clients, de laisser des traces de leur activité à destination d'autrui dans le système d'information. Lorsque cet autrui est connu du conseiller client, non personnellement, mais du point de vue de l'étape qui 
suit et du métier associé, le conseiller client «transmet » des éléments qu'il sait utiles lors de la prochaine étape. Lorsque cette prochaine étape n'est pas identifiée, et donc que le conseiller client n'a pas idée des personnes qui auront à reprendre ultérieurement le traitement de cette demande, il «met en archives» des éléments qu'il pense pouvoir être utiles pour l'avenir du traitement de la demande.

\subsection{Apports croisés de l'approche instrumentale et du cours d'action pour approcher les phénomènes transitionnels}

Sans le cours d'action, nous n'aurions pas été en mesure d'appréhender la dimension phénoménologique de l'activité, sans appréhender la dimension phénoménologique, nous n'aurions pas été en mesure d'appréhender la dimension sémiologique, le sens de l'activité pour le sujet, sans la dimension sémiologique, nous n'aurions pu rendre compte de phénomènes transitionnels des activités narratives; puisque les sujets tissent de la continuité plus profonde pour eux même. Bien que cette continuité puisse s'apparenter à de la discontinuité extérieure, pour un observateur extérieur, Il s'agit bien de comprendre pour le chercheur les formes de continuités plus profondes.

Sans l'approche instrumentale, nous n'aurions pas été en mesure d'appréhender la dimension matérielle et instrumentale de l'activité narrative comme nous l'avons fait. Nous n'aurions pas pu cumuler des épisodes d'activité instrumentées pour repérer les instruments transitionnels dans une maille diachronique.

Sans la conjonction de ces deux approches, nous n'aurions pu rendre compte des mouvements de fusion/dissociation, qui sont les phénomènes qui permettent de dépasser les frontières pour le sujet. Nous n'aurions pu rendre compte de ce qui émerge, se réifie, se refluidifie dans l'entrelacs des mondes, car c'est bien ce que nous traquions, autrement dit, nous aurions échoué à attraper les dynamiques développementales encapsulées dans ces activités narratives transitionnelles.

\subsection{Différentes mailles de transition, de franchissement de frontières}

67 La comparaison de ces deux recherches montre que l'articulation des cadres du cours d'action et de l'approche instrumentale permet d'appréhender des transitions à différentes mailles.

Ainsi, dans la sphère du récit de voyage, il s'agit de transitions appréhendées à la maille de l'individu. Les instruments transitionnels mis en évidence dans cette recherche sont des ressources que le sujet crée pour le soi en devenir, qui permettent de créer de la continuité du côté du sujet afin de garder un lien avec l'expérience de voyage tout en pouvant la considérer comme extérieure à soi. Les instruments transitionnels permettent ici de penser dans le même temps des activités narratives qui sont de nature différente selon les contextes successifs : lors du recueil, les activités narratives sont synchrones et intriquées avec d'autres activités variées (rencontre de personnes, déplacements), elles permettent au narrateur du voyage de s'extraire d'une situation englobante. En revanche, elles sont au premier plan lors de l'élaboration du récit, le narrateur se retrouve en situation de visu avec ses matériaux qu'il consulte pour revisiter son expérience et construire un récit. Ces instruments transitionnels révèlent 
les diverses formes d'activités à l'œuvre au sein d'activités narratives successives ainsi que leurs réifications successives.

Dans le cadre d'un processus commercial de relation clientèle, il s'agit de transitions appréhendées selon une maille collective transverse, à l'articulation entre les niveaux collectif et organisationnel. Les instruments transitionnels mis en évidence dans cette recherche sont en effet des ressources qui permettent de créer la continuité de l'activité collective transverse, et ce faisant la continuité du traitement de la demande $\mathrm{du}$ client. Ces instruments rassemblent des fragments du système technicoorganisationnel au service d'une activité collective qui fonde la transversalité au sein des organisations. L'approche transitionnelle permet ainsi d'accéder au niveau de l'organisation de manière intrinsèque, en partant de l'individu pour remonter jusqu'au niveau organisationnel. Un des prochains objectifs de notre programme de recherche autour de cette approche est de la mettre en œuvre jusqu'au niveau interorganisationnel, dans le cadre de supply chains.

C'est l'articulation des deux cadres conceptuels qui nous permet de retisser la continuité sémiologique et structurelle d'unités d'activités développementales instrumentées et distribuées dans des espaces-temps différents dans la sphère du récit de voyage d'une part ainsi que dans le contexte d'un processus commercial de relation clientèle d'autre part. Nous qualifions ces activités développementales qui s'élaborent dans un mouvement de la durée et du devenir «des activités transitionnelles instrumentées». Dans cette perspective, le cours d'action nous permet d'analyser le décours temporel singulier continu et discontinu des activités transitionnelles tandis que l'approche instrumentale nous permet d'opérer des découpes structurelles d'activités transitionnelles instrumentées. En adoptant une unité d'analyse diachronique et sémiologique qui englobe et permet de faire tenir ensemble des activités hétérogènes se déroulant dans des espaces temps différents, ainsi qu'en examinant non seulement la succession des séquences d'activités transitionnelles instrumentées, mais également la succession des séquences d'activités sémiologiques, qui font signe de la même manière pour les acteurs, mais dans des espaces-temps différents, nous sommes à même de caractériser une phénoménologie des activités transitionnelles, autrement dit une phénoménologie des activités en train de se transformer selon une dynamique développementale.

\section{Conclusion}

71 Cet article explicite comment l'articulation du cours d'action et de l'approche instrumentale a permis de faire émerger une approche transitionnelle, qui met le focus sur le franchissement de frontières d'un point de vue intrinsèque. Deux recherches en ergonomie contrastées du point de vue du domaine d'activité ont constitué les prémisses de cette approche. Ces recherches ont mis en évidence des instruments dits transitionnels, c'est à dire des instruments qui permettent de maintenir une continuité de l'expérience/d'un processus malgré la discontinuité qui caractérise les activités étudiées. L'approche transitionnelle cherche à se déprendre d'une position en surplomb pour accompagner le pouvoir d'agir des sujets, en repartant de leurs propres genèses, de la manière dont ils bricolent des instruments transitionnels pour faire grandir et déployer leur activité narrative. Orienter la focale sur le sujet capable 
(Rabardel, 2005) consiste à respecter et encourager les chemins singuliers de développements de sujets et des collectifs de travail.

Nous avons exposé en filigrane au fil de cet article la manière dont nous avons concilié le cadre théorique du cours d'action développé par Theureau (2004) ; ainsi que le cadre théorique de l'approche instrumentale développé par Rabardel $(1995,2005)$ pour se donner les moyens, à la manière d'un photographe, de traquer les phénomènes transitionnels qui nous intéressent en variant les focales pour ne pas occulter les différentes échelles/granularités impliquées dans le développement d'un sujet en devenir ou d'un collectif de travail transverse pour franchir les frontières d'une organisation induite par le système d'information. Nous qualifions ces activités de franchissement de frontières d' " activités transitionnelles ». Ainsi, pour accompagner le développement des organisations, nous soutenons qu'une approche transitionnelle est nécessaire. Les partis pris d'une telle approche sont tout autant épistémiques que pragmatiques :

1. Au plan épistémique, il s'agit d'identifier les systèmes d'activité et les personnes-frontières qui œuvrent aux frontières de ces systèmes d'activité afin d'analyser l'activité transitionnelle, c'est à dire la manière dont ces personnes tissent de la continuité pour faire tenir ensemble ces systèmes hétérogènes pour elles-mêmes dans un premier temps, ce qui peut leur permettre dans un second temps de raccorder les mondes. C'est cette activité individuelle et collective qui consiste à tisser de la continuité et articuler plusieurs systèmes d'activité que nous qualifions de transitionnelle. L'analyse de l'activité des personnesfrontières permet de comprendre les logiques hétérogènes auxquelles elles se heurtent ainsi que les stratégies et ressources élaborées pour dépasser ces logiques parfois contradictoires et les faire tenir ensemble, pour franchir les frontières organisationnelles.

Plus précisément, l'approche transitionnelle cherche à appréhender la manière dont les personnes franchissent des frontières, en tissant de la continuité entre les mailles individuelles, collectives, organisationnelles et inter-organisationnelles, qu'elles occupent des fonctions explicitement tournées vers le franchissement de frontières ou non. Dans les organisations, certaines fonctions sont en effet prévues pour articuler et coordonner des mondes différents. C'est le cas par exemple des pilotes de processus, des « gestionnaires de cas» (Ennuyer, 2012) dans le domaine de la santé, et autres professions dont le cœur consiste à faire de la coordination. Ces personnes peuvent extrinsèquement être désignées comme des personnes-frontières, ce qui n'est pas le cas des voyageurs et des conseillers clients. Il est en effet important de regarder les franchissements de frontière réalisés par des personnes qui n'ont pas une fonction explicitement dédiée à cela, mais qui réalisent ces franchissements dans le cadre d'activités transitionnelles ordinaires.

2. Au plan pragmatique de l'intervention, il convient de préciser que dans une perspective transitionnelle, l'ergonome devient nécessairement personne-frontière, en contribuant par son analyse à identifier les stratégies de franchissement de frontières des sujets, il participe à son tour à favoriser la recomposition d'unités d'activités fragmentées par l'intermédiaire de dispositifs de réflexivité d'une part (entretiens d'autoconfrontation), et par l'animation et la co-conception de groupes de travail incluant des acteurs issus de systèmes d'activités diversifiés et contradictoires, d'autre part (Folcher, 2015).

73 Au-delà des instruments transitionnels, l'approche transitionnelle pourrait-elle appréhender (examiner, accompagner, co-concevoir) des dispositifs transitionnels au sein des organisations? Comme le dit très justement Pastré (2011), avec le développement d'une ontologie et d'une épistémologie du sujet capable, l'accent se déplace, là où les théories de l'activité reposent sur une distinction entre la sphère du sujet et la sphère de l'objet de l'activité, c'est désormais un couplage entre les sujets et 
le monde qu'il s'agit de décrypter. Il s'agit de penser un sujet qui s'engage dans le monde pour le transformer et se transformer lui-même. La proposition de l'approche transitionnelle est d'examiner ce qui se passe au cours des épisodes transitionnels au sein desquels se réorganise l'expérience du sujet ou d'un collectif de sujet(s) via un mouvement d'union et de séparation du sujet et du monde. Nous soutenons que le développement peut advenir à différentes échelles dans l'intervalle de l'épisode transitionnel. Autrement dit l'approche transitionnelle examine plus précisément les reconfigurations intrinsèques qui adviennent dans le rapport des mailles locale et globale de l'activité. Mais qu'est-ce qui s'élabore dans ce mouvement du devenir? Comment faire tenir ensemble des mondes hétérogènes? Comment reconstituer des unités d'activité fragmentées, brisées par les frontières organisationnelles? Comment soutenir et décrypter des genèses qui adviennent à différentes échelles d'une organisation: genèses instrumentales (Rabardel, 1995) genèses identitaires (Pastré, 2005), genèses professionnelles (Béguin, 2005) ? C'est l'ensemble des questions ouvertes par le chantier conceptuel et empirique qui tente d'appréhender la thématique des genèses organisationnelles (Folcher, \& Bationo, 2018).

\section{BIBLIOGRAPHIE}

Akkerman, S., \& Bakker, A. (2011). Boundary Crossing and Boundary Objects. Review of Educational Research, 81(2), 132-169.

Bationo Tillon, A. (2006). Pratiques des activités narratives instrumentées : Une analyse diachronique et structuro-fonctionnelle en amont de la conception. Doctorat d'ergonomie, Saint Denis : Université de Paris 8.

Bationo-Tillon, A. (2017). Chemin transitionnel - ouverture pluridisciplinaire pour une ergonomie développementale. Habilitation à diriger des recherches, Université Paris 8.

Bationo-Tillon, A., Folcher, V., \& Rabardel, P. (2010). Les instruments transitionnels : une proposition pour étudier la diachronie des activités narratives. Activités [En ligne], 7-2.

Bationo-Tillon, A., \& Rabardel, P. (2015). L'approche instrumentale : conceptualiser et concevoir pour le développement. In F. Decortis (Ed.), L'ergonomie orientée enfants. Concevoir pour le développement (pp. 109-145). Paris : PUF.

Béguin (2005) Concevoir pour les genèses professionnelles ; p.31-52. In P. Rabardel \& P. Pastré (Eds.), Modèles du sujet pour la conception (pp. 231-260). Toulouse : Octarès.

Bouvier, N. (1992). Usage du monde. Paris : Payot.

Brulhart, F., \& Favoreu, C. (2006). Le lien contrôle-confiance-performance dans les relations de partenariat logistique inter-firmes. Finance Contrôle Stratégie, 9(5), 59-96.

Bruner, J.-S. (1990). Acts of meanings. Cambridge : Havard University Press.

Christin, R. (2000). L'imaginaire voyageur ou l'expérience exotique. Paris : Éditions L'Harmattan. 
Engestrom, Y. (2001). Expansive learning at work: Toward an activity theoretical reconceptualization. Journal of Education and Work, 14, 133-156.

Ennuyer, B. (2012). Un regard critique sur les « gestionnaires de cas », dernière « mode » de la coordination imposée par le plan Alzheimer. Gérontologie et société, 35(142), 205-213.

Fawcett, S., \& Magnan, G. (2002). The rhetoric and reality of supply chain integration. International Journal of Physical Distribution \& Logistics Management, 32(5), 339-361.

Folcher V. (2015). Concevoir pour et dans l'usage, la maitrise d'usage en conduite de projet, Revue des Interactions Humaines Médiatisées, RIHM, 16(1), 39-60.

Folcher V., \& Bationo-Tillon A. (2018). Approche instrumentale dans l'intervention ergonomique. Congrès de la SELF 2018. Bordeaux.

Folcher, V., \& Rabardel, P. (2004). Hommes, Artefacts, Activités : perspective instrumentale. In P. Falzon (Ed.), Ergonomie (pp. 251-268). Paris : PUF.

Hutchins, E. (1995). Cognition in the wild. Cambridge : MIT Press, 381 p.

Maturana, H., \& Varela, F. (1980). Autopoiesis and Cognition. Dordrecht : Reidel.

Motté, F., \& Haradji, Y. (2010). Construire la relation de service en considérant l'activité humaine dans ses dimensions individuelles et collectives. In G. Valléry, M.-C. Le Port, \& M. Zouinar (Eds.), Ergonomie, conception de produits et services médiatisés (pp. 11-35). Paris : PUF.

Motté, F., \& Poret, C. (2018). La Simulation Réflexive Transvserse : une méthode pour ancrer l'activité humaine au cœur de la performance de l'entreprise. Activités, 15(1).

Pastré, P. (2005) Genèse et identité. In P. Rabardel \& P. Pastré (Eds.), Modèles du sujet pour la conception (pp. 231-260). Toulouse : Octarès.

Pastré, P. (2011). La didactique professionnelle. Approche anthropologique du développement des adultes. Paris : PUF.

Poret, C. (2015). Concevoir pour le Pouvoir d'agir ensemble d'un collectif transverse. Le cas de la relation de service dans le domaine commercial. Thèse de doctorat, Université Paris VIII.

Poret, C., Folcher, V., Motté, F., \& Haradji, Y. (2016). Concevoir pour le Pouvoir d'agir ensemble au sein des organisations : le cas d'un processus commercial. Activités, 13(2).

Rabardel, P. (1995). Les Hommes et les technologies. Paris : Armand Colin.

Rabardel, P. (2001). Instrument mediated activity in situations. In A. Blandford \&

P. Vanderdonckt (Eds.), People and computers XV Interactions without frontiers (pp. 17-30). Verlag : Springer.

Rabardel, P. (2005). Instrument subjectif et développement du pouvoir d'agir. In P. Rabardel, \& P. Pastré (Eds.), Modèles du sujet pour la conception. Dialectiques activités développement (pp. 11-29). Toulouse : Octarès.

Rabardel, P., \& Béguin, P. (2005). Instrument mediated activity: from subject development to anthropocentric design. Theoretical Issues in Ergonomics Science, 6(5), 429-461.

Rabardel, P., \& Pastré, P. (2005). Modèles du sujet pour la conception. Dialectiques activités développement. Toulouse : Octarès.

Rix, G., \& Biache, M-J. (2004). Enregistrement en perspective subjective située et entretien en resitu subjectif : une méthodologie de constitution de l'expérience. Intellectica, n 38, 363-396. 
Suchman, L. (1987). Plans and Situated Actions - The Problem of Human/Machine Communication. New York : Cambridge university press.

Theureau, J. (2004). Le cours d'action : méthode élémentaire. Toulouse : Octarès.

Theureau, J. (2006). Le cours d'action : méthode développée. Toulouse : Octarès.

Vermersch, P. (1994). L'entretien d'explicitation. Issy Les moulineaux : ESF.

Wenger, E. (1998). Communities of practice, learning, meaning and identity. Cambridge, UK :

Cambridge University Press.

Winnicott, D.-W. (1971). Objet transitionnels et phénomènes transitionnels, Jeu et réalité. Paris :

Gallimard.

\section{NOTES}

1. Par mondes du sujet, nous définissons le terme de monde selon la logique intrinsèque des sujets. Le ou les mondes des sujets correspondent à des espaces-temps subjectivo-centrés. La pluralité des mondes du sujet correspond à la pluralité des manières d'être au monde de ce sujet. 2. Seules les trois premières étapes sont communes aux deux études. La quatrième étape est spécifique à celle relative aux voyageurs.

3. Nous nous étions mis d'accord avec les participants de l'étude et une tierce personne participant à l'expédition polaire pour que soient enclenchés des enregistrements vidéos de l'activité narrative aux moments opportuns (lors de l'expédition, en soirée sous la toile de tente ; au retour lorsque les narrateurs s'attelaient à l'élaboration de récits).

4. Chacune de ces U.S.E.s a, pour soubassement, un signe dit hexadique dans la mesure où il relie, dans une structure relationnelle précise, six composantes (Engagement-Actualité potentielleRéférentiel-Représentamen- Use- Interprétant) qui sont supposées résumer les processus en jeu dans une unité d'activité significative pour l'acteur.

5. Signe tétradique : le signe tétradique a été développé antérieurement au signe hexadique dans l'élaboration théorique de Theureau, il est composé de quatre composantes (objet, représentamen, interprétant, use)

6. Pour emprunter le langage du cours d'action, il s'agit de ce qui fait signe pour l'acteur. Le Representamen (R) est un élément du signe hexadique qui est proche de la notion de "perturbation» de Varela. Dans l'approche du cours d'action, les Representamen sont des perturbations qui donnent lieu à conscience préréflexive.

7. Dans l'approche du cours d'action, les ouverts constituent les dimensions structurelles de l'Engagement de l'acteur dans la situation, en ouvrant un horizon d'attente. L'ensemble des ouverts, qui ont été créés dans le passé mais qui ne sont pas encore refermés constituent le système des ouverts à l'instant $t$, cadre d'interprétation des perturbations ou évènements pour l'acteur. 


\section{RÉSUMÉS}

Nous proposons au sein de cet article d'aborder la question des voies conceptuelles possibles pour penser les organisations en devenir. Nous commencerons par introduire le concept de frontière qui nous semble heuristique pour définir le développement des organisations comme franchissement de frontières. Nous proposerons dans un second temps d'aborder le franchissement de frontière d'un point de vue intrinsèque à travers le prisme transitionnel. Pour rendre compte de ces phénomènes transitionnels, nous avons hybridé deux cadres conceptuels de l'ergonomie que sont le cours d'action et l'approche instrumentale. À travers deux recherches contrastées du point de vue des domaines d'activité (narrations de voyage et un processus de traitement de la demande client dans le champ de l'énergie), nous confronterons la manière dont il est possible d'hybrider ces deux cadres théoriques conceptuellement, méthodologiquement et empiriquement. Nous mettrons en évidence ce qui résulte de cette hybridation : des instruments dits transitionnels porteurs de continuité à différentes échelles. Enfin, nous évoquerons les perspectives ouvertes par une approche transitionnelle pour penser les genèses organisationnelles.

In this paper, we propose to address the question of possible conceptual ways of thinking about organizations in the making. We begin by introducing the concept of boundary, which we feel to be heuristic when defining development as a crossing of borders. We then propose to consider boundary crossing from an intrinsic point of view by adopting a transitional prism. To address these transitional phenomena, we articulate two conceptual frameworks of ergonomics: course of action and the instrumental approach. Through two works of research that differ in terms of fields of activity (narrations of travel and a process for dealing with customer requests in the field of energy), we compare ways of conceptually, methodologically and empirically hybridizing these two theoretical frameworks. We highlight what results from this hybridization: transitional instruments that channel continuity on different scales. Finally, we discuss the perspectives that a transitional approach offers to think about organizational genesis.

\section{INDEX}

Mots-clés : approche transitionnelle, développement, frontières, genèse organisationnelle

Keywords : transitional approach, development, organizational genesis, boundaries

\section{AUTEURS}

\section{ANNE BATIONO-TILLON}

Université Paris 8/laboratoire Paragraphe/équipe C3U, 2 rue de la liberté, 93526 Saint-Denis

Cedex - anne.bationo-tillon@univ-paris8.fr

\section{CÉLINE PORET}

Institut de Radioprotection et de Sûreté Nucléaire, Laboratoire des Sciences Humaines et Sociales (LSHS), B.P. 17 - 92262 Fontenay-aux-Roses Cedex - celine.poret@irsn.fr 


\section{VIVIANE FOLCHER}

Université Paris 8/laboratoire Paragraphe/équipe CITU, 2 rue de la liberté, 93526 Saint-Denis Cedex - viviane.folcher@univ-paris8.fr 